Honam Mathematical J. 36 (2014), No. 1, pp. 085-102

http://dx.doi.org/10.5831/HMJ.2014.36.1.85

\title{
GRAPHICAL ARRANGEMENTS OF COMPRESSED GRAPHS
}

\author{
Thi A. Nguyen and SAngwook KIM*
}

\begin{abstract}
We show that if a graph $G$ is compressed, then the proper part of the intersection poset of the corresponding graphical arrangement $A_{G}$ has the homotopy type of a wedge of spheres. Furthermore, we also indicate the number of spheres in the wedge, based on the number of adjacent edges of vertices in $G$.
\end{abstract}

\section{Introduction}

A hyperplane arrangement $\mathcal{A}$ is a finite set of affine hyperplanes in some vector space $V \cong K^{n}$, where $K$ is a field. There are a lot of examples of hyperplane arrangements in $K^{n}$ such as braid arrangements, Shi arrangements, and Catalan arrangements.

Graphical arrangements are special hyperplane arrangements obtained from finite simple undirected graphs. They are studied in the connection with graph theory $[5,9]$. Many of the invariants associated with a graphical arrangement can be computed directly from the corresponding graph. For example, the characteristic polynomial of a graphical arrangement is the chromatic polynomial of the corresponding graph (see [8, section 2.4], [10]). The braid arrangement $B_{n}$ is the graphical arrangement of the complete graph $K_{n}$. We also know that the graphical arrangement of a general graph $G$ on vertex set $[n]:=\{1,2, \ldots, n\}$ is a subarrangement of the braid arrangement $B_{n}$.

The intersection poset $L(\mathcal{A})$ of a hyperplane arrangement $\mathcal{A}$ is the set of all nonempty intersections of hyperplanes in $\mathcal{A}$, including $V$ itself

Received November 29, 2013. Accepted January 15, 2014.

2010 Mathematics Subject Classification. 05C88, 05 C89.

Key words and phrases. compressed graph, graphical arrangement.

This research was supported by Basic Science Research Program through the National Research Foundation of Korea(NRF) funded by the Ministry of Education, Science and Technology(NRF-2012R1A1A1013149).

${ }^{*}$ Corresponding author 
as the intersection over the empty set, ordered by reverse inclusion. The intersection poset is an important invariant of the arrangement. The number of regions and bounded regions of a real hyperplane arrangement is determined by its intersection poset [13] and the Betti numbers of the complement of a complex hyperplane arrangement can be expressed using the intersection lattice [7]. The topological property of the proper part of the intersection poset is related to its order complex, where the order complex $\Delta(P)$ of a poset $P$ is the abstract simplicial complex whose vertices are all elements of $P$ and whose simplices are all finite chains of $P$, including the empty chain. A property of a poset studied a lot is shellability $[3,4]$. A shellable poset has the homotopy type of a wedge of spheres ([3, theorem 4.1]). One nice way to show the shellability of a poset is the lexicographic shellability $[1,2,3,4,6]$. This method also provides the number of spheres in the wedge by counting decreasing maximal chains in the intersection poset.

It is well-known that the intersection poset of the braid arrangement $B_{n}$ is isomorphic to the partition lattice $\Pi_{n}$ of the $n$-element set ordered by refinement $\left[8\right.$, proposition 2.9]. Since $\bar{\Pi}_{n}$ is known to be EL-shellable $[1,11]$, one can compute the homotopy type of the proper part of the intersection poset of the braid arrangement $B_{n}$ (see [12]). We will show this result again by using the number of decreasing maximal chains.

In this paper, we study the topological property of the graphical arrangement of a compressed graph $([4$, p.3952]). It is known that compressed graphs are supersolvable, and the intersection poset of supersolvable graphs are $E L$-shellable ([9]). In this paper, we provide a new $E L$-shelling which can be easily obtained from graphs. Furthermore, we also describe the number of spheres in the wedge, based on adjacent edges of vertices in $G$.

The rest of the paper is organized as follows. Section 2 provides the basic notions and definitions which will be used throughout the paper. In Section 3, an edge-labeling is defined and shown to be an EL-labeling. The last section describes the number of spheres in the wedge using the number of decreasing chains. The enumerative results for special cases will be given as well.

\section{Basic notions and definitions}

Let $G$ be a simple undirected graph on a vertex set $[n]$ and $E(G)$ denote the set of edges of $G$. We will use the notation $i j$ for an edge of 
$G$ with two vertices $i$ and $j$. Let $V=K^{n}$ be a vector space over a field $K$ with coordinates $x_{1}, x_{2}, \ldots, x_{n}$.

Definition 2.1. The graphical arrangement $\mathcal{A}_{G}$ in a vector space $V$ is the hyperplane arrangement

$$
\left\{x_{i}-x_{j}=0 \mid i j \in E(G)\right\}
$$

In the intersection poset of the graphical arrangement $\mathcal{A}_{G}$, we also label each hyperplane $x_{i}-x_{j}=0$ by $i j$. There are two cases to label an intersection of two hyperplanes. If two hyperplanes are $i j$ and $k l$, where $\{i, j\} \cap\{k, l\}=\emptyset$, then the common subspace is labeled by $i j-k l$. Otherwise, we may assume that $l=j$. Then the common subspace will be labeled by $i j k$. A general subspace in the intersection poset is denoted by $A=A_{1}-A_{2}-\cdots-A_{r}$, where $A_{i}, i=1,2, . ., r$, are the sets of indices of coordinates. We call $A_{i}, i=1,2,3, \ldots, r$, blocks of $A$, and those indices of coordinates indices of $A$. When we get the intersection of two subspaces $H=H_{1}-H_{2}-\cdots-H_{p}$ and $K=K_{1}-K_{2}-\cdots-K_{q}$, where $H_{i}$ and $K_{i}$ are blocks of $H$ and $K$, we also have two cases to label their common subspace. If there is a common index in two blocks $H_{i}$ of $H$ and $K_{j}$ of $K$, then in the common subspace we get the union of $H_{i}$ and $K_{j}$ as union of two sets. Otherwise, the common space is denoted by $H_{1}-H_{2}-\cdots-H_{p}-K_{1}-K_{2}-\cdots-K_{q}$. Furthermore, we say $K<H$ if each block $K_{j}$ of $K$ is contained in some block $H_{i}$ of $H$.

Example 2.2. Consider a graph $G_{1}$ shown in Figure 1. The graphical arrangement $\mathcal{A}_{G}$ of $G$ consists of hyperplanes $x_{1}-x_{3}=0, x_{1}-x_{4}=0$, and $x_{2}-x_{5}=0$. In the intersection poset of $\mathcal{A}_{G}$, we label hyperplane $x_{1}-x_{3}=0$ by $13, x_{1}-x_{4}=0$ by 14 , and $x_{2}-x_{5}=0$ by 25 . Figure 1 shows the intersection poset $P_{G_{1}}$ of $\mathcal{A}_{G}$.

Definition 2.3. A simple undirected graph $G$ on a vertex set $[n]$ is compressed if $i<j<k$ and $i k \in E(G)$ imply that $i j \in E(G)$.

Example 2.4. Complete graphs $K_{n}$ are compressed graphs. Paths $P_{n}$ with the edges $\{i, i+1\}$ for all $i=1,2, \ldots, n-1$ are compressed graphs. Furthermore, Figure 2 shows other examples of compressed and noncompressed graphs.

Definition 2.5. A partially order set $P$ (or poset, for short) is a set together with a relation $\leq\left(\right.$ or $\left.\leq_{P}\right)$ satisfying the following three axioms:

- For all $t \in P, t \leq t$.

. If $s \leq t$ and $t \leq s$, then $s=t$.

. If $s \leq t$ and $t \leq u$, then $s \leq u$. 


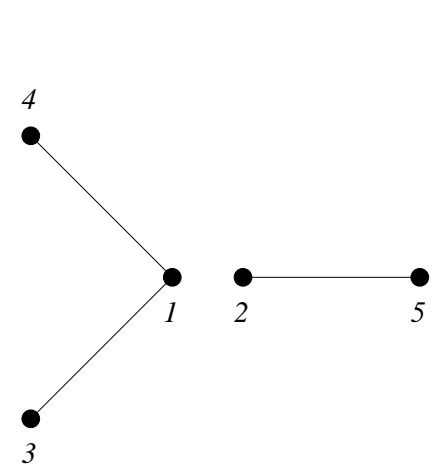

$G_{1}$

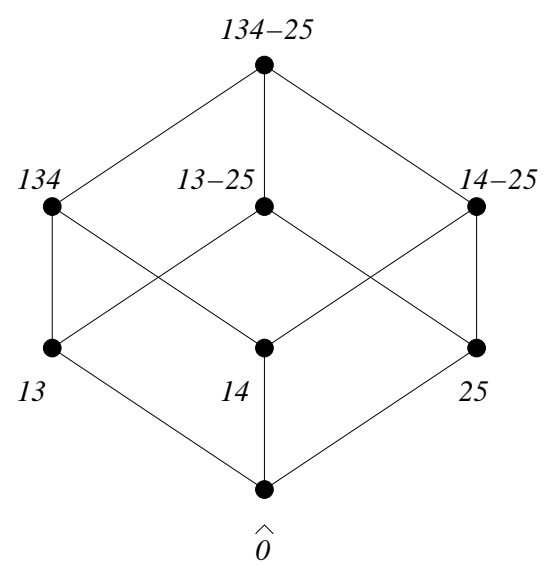

$P_{G_{I}}$

Figure 1. A graph $G_{1}$ and the intersection poset $P_{G_{1}}$

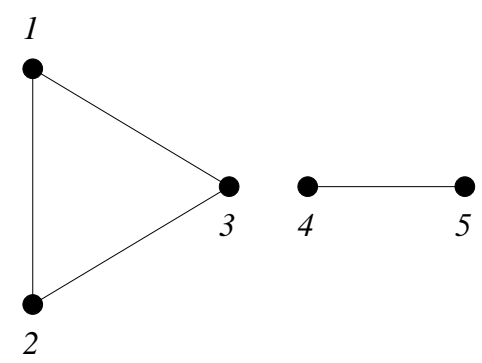

$G_{2}$

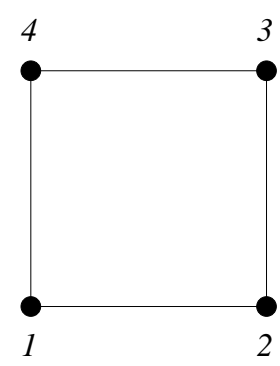

$G_{3}$

Figure 2. A compressed graph $G_{2}$ and a noncompressed graph $G_{3}$

For $s, t \in P$, the (closed) interval $[s, t]=\{u \in P: s \leq u \leq t\}$. We say that $t$ covers $s$ or $s$ is covered by $t$ (denote $s \lessdot t$ ), if $s<t$ and no element $u \in P$ satisfies $s<u<t$. $P$ has a $\hat{0}$ if there exists an element $\hat{0} \in P$ such that $t \geq \hat{0}$ for all $t \in P$. Similarly, $P$ has $a \hat{1}$ if there exists an element $\hat{1} \in P$ such that $t \leq \hat{1}$ for all $t \in P$. A poset is bounded if it contains both $\hat{0}$ and $\hat{1}$. The Hasse diagram of a finite poset $P$ is the graph whose vertices are the elements of $P$, whose edges are the cover relations, and 
such that if $s<t$ then $t$ is drawn above $s$ (i.e., with a higher vertical coordinate). See $P_{G_{1}}$ as Figure 1 for the Hasse diagram of a poset. A chain is a poset in which any two elements are comparable. A subset $C$ of a poset $P$ is called a chain if $C$ is a chain when regarded as a subposet of $P$. The chain $C$ of $P$ is called maximal if it is not contained in a larger chain of $P$. The length $l(C)$ of a finite chain is defined by $\|C\|-1$. The length of a finite poset $P$ is $l(P):=\max \{l(C): C$ is a chain of $P$ \}.

Definition 2.6. An edge-labeling of a poset $P$ is a map $\lambda: \mathcal{E}(P) \rightarrow \Lambda$, where $\mathcal{E}(P)$ is the set of edges of the Hasse diagram of poset $P$ and $\Lambda$ is an ordered set. For an edge $\{s, t\} \in \mathcal{E}(P)$, we denote $\lambda(s, t)$ for $\lambda(\{s, t\})$.

Definition 2.7. An edge-labeling is called an $E L$ - labeling (edge lexicographical labeling) if for every interval $[x, y]$ in poset $P$, there is a unique increasing maximal chain, which lexicographically precedes all other maximal chains of $[x, y]$.

Björner and Wachs [3] showed the following fundamental result about the EL-labeling.

Theorem 2.8. If a bounded poset $P$ admits an $E L$-labeling, then the order complex $\Delta(\bar{P})$ has the homotopy type of a wedge of spheres. Furthermore, the number of $i$-spheres is the number of decreasing maximal $(i+2)$-chains of $P$.

\section{The edge-labeling}

In this section, we will define an edge-labeling of the intersection poset of the graphical arrangement for a compressed graph, and show that it is an EL-labeling.

Let $P_{G}$ be the intersection poset of the graphical arrangement $\mathcal{A}_{G}$ of a compressed graph $G$. We now describe an edge-labeling $\lambda: \mathcal{E}\left(P_{G}\right) \rightarrow$ $(\mathbb{Z}, \mathbb{Z})$, where $(\mathbb{Z}, \mathbb{Z})$ is a partially ordered set with the order defined by $(a, b)<(c, d)$ if $a<c$ or $a=c$ and $b<d$. We can identify $(a, 0) \in(\mathbb{Z}, \mathbb{Z})$ with $a \in \mathbb{Z}$.

Let $H$ and $K$ be two elements in $P_{G}$ where $H$ covers $K$. There are three kinds of coverings in $P_{G}$ which are described in the following labeling rule:

Case 1: when $H=H_{1}-\cdots-H_{i} x-\cdots-H_{m}$ and $K=H_{1}-\cdots-H_{m}$ for some $i(1 \leq i \leq m)$

$$
\lambda(K, H):=\min \left\{(x, y) \mid x y \in E(G) \text { and } y \in H_{i}\right\}
$$




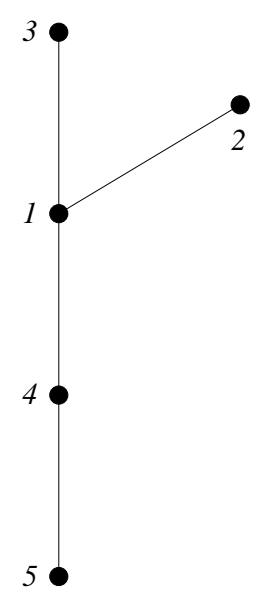

$G_{4}$

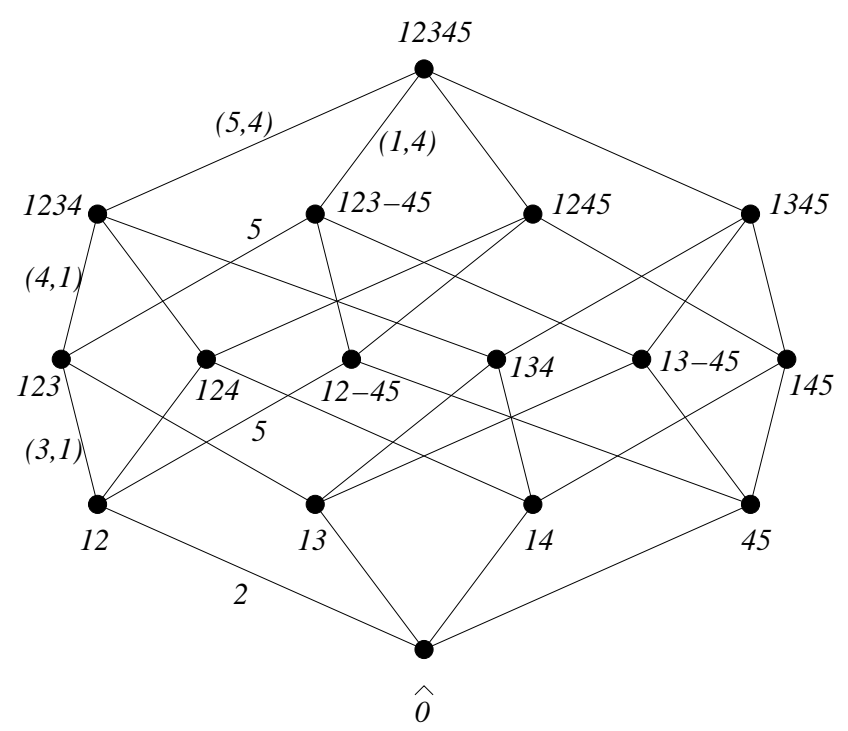

$P_{G_{4}}$

Figure 3. A compressed graph $G_{4}$ and the intersection poset $P_{G_{4}}$

Case 2: when $H=H_{1}-\cdots-H_{m}-x y$ and $K=H_{1}-\cdots-H_{m}$

$$
\lambda(K, H):=\max \{x, y\}
$$

Case 3: when $H=H_{1}-\cdots-H_{i} \cup H_{j}-\cdots-H_{m}$ and $K=H_{1}-\cdots-$ $H_{i}-\cdots-H_{j}-\cdots-H_{m}$ for some $i, j(1 \leq i<j \leq m)$

$\lambda(K, H):=\min \left\{(u, v) \mid u v \in E(G)\right.$ and $u \in H_{i}, v \in H_{j}$ or $\left.v \in H_{i}, u \in H_{j}\right\}$

Example 3.1. Figure 3 shows an example of compressed graph $G_{4}$ and the intersection poset $P_{G_{4}}$ with some labeling values. In the poset $P_{G_{4}}, 12$ is covered by 123 , and the labeling value $\lambda(12,123)$ is given by Case 3. When we find the labeling value for the edge $\{K, H\}$ where $K=\hat{0}$, we use Case 3 to label that edge. And the last case is used to find the labeling value of the edge $\{123-45,12345\}$ of the example.

Let $M$ and $N$ be two elements in the poset $P_{G}$ such that $N<M$.

Lemma 3.2. There exists an increasing maximal chain from $N$ to $M$. 
Proof. Assume that $N=N_{1}-N_{2}-\cdots-N_{p}$ and $M=M_{1}-M_{2}-\cdots-$ $M_{q}$. A covering element of $N$ in $[N, M]$ must have one of the following forms:

(Form 1) $N_{1}-N_{2}-\cdots-N_{i} x-N_{p}$, or

(Form 2) $N_{1}-N_{2}-\cdots-N_{p}-x y$, or

(Form 3) $N_{1}-N_{2}-\cdots-N_{i} \cup N_{j}-\cdots-N_{p}$.

Let $A_{1}:=N_{1}-N_{2}-\cdots-N_{i} x-N_{p}$ where $\lambda\left(N, A_{1}\right)$ is minimal among all covering elements of $N$ of the form 1 . There is only one element $A_{1}$ of form 1 satisfying this condition.

Let $A_{2}:=N_{1}-N_{2}-\cdots-N_{p}-x y(x<y)$, where $\lambda\left(N, A_{2}\right)=y$ is minimal among all covering elements of $N$ of the form 2. Assume that there exists $A_{2}^{\prime}=N_{1}-N_{2}-\cdots-N_{p}-x^{\prime} y\left(x^{\prime}<y\right)$, where $N \lessdot A_{2}^{\prime}<M$ and $\lambda\left(N, A_{2}^{\prime}\right)=\lambda\left(N, A_{2}\right)=y$. Since $x<y$ and $x^{\prime}<y$, we have $x<x^{\prime}<y$ or $x^{\prime}<x<y$. Since $G$ is compressed, there exists edge $x x^{\prime}$. Therefore, we have another element $A_{2}^{\prime \prime}=N_{1}-N_{2}-\cdots-N_{p}-x x^{\prime}$ satisfying $N \lessdot A_{2}^{\prime \prime}<M$. Since $\lambda\left(N, A_{2}^{\prime \prime}\right)=x$ or $x^{\prime}$ and both of these are less than $y$, this contradicts the condition of $A_{2}$. So there exists only one element of form 2 that satisfies the condition.

Let $A_{3}:=N_{1}-N_{2}-\cdots-N_{i} \cup N_{j}-\cdots-N_{p}$ where $\lambda\left(N, A_{3}\right)=(u, v)$ is minimal among all covering elements of $N$ of the form 3 . We also have only one element $A_{3}$ of form 3 satisfying the above condition.

Choose the covering element $N_{1}$ of $N$ so that

$$
\lambda\left(N, N_{1}\right)=\min \left\{\lambda\left(N, A_{1}\right), \lambda\left(N, A_{2}\right), \lambda\left(N, A_{3}\right)\right\} .
$$

Since $\lambda\left(N, A_{1}\right), \lambda\left(N, A_{2}\right)$, and $\lambda\left(N, A_{3}\right)$ are pairwise distinct, we have only one choice of $N_{1}$. We find the covering element of $N_{1}$ in the similar way with finding the covering element of $N$. Continue this process until we get

$$
\mathcal{C}:=N \lessdot N_{1} \lessdot \cdots \lessdot N_{\ell}=M .
$$

See Example 3.5 for the choosing.

Now we claim that $\mathcal{C}$ is an increasing chain.

Let $A, B$, and $C$ be three elements in $\mathcal{C}$ satisfying $A \lessdot B \lessdot C$. It is enough to show that $\lambda(A, B)<\lambda(B, C)$. Assume that $A$ has form $A=A_{1}-A_{2}-\cdots-A_{k}$. The proof is divided into small cases based on the form of $B$ and $C$. Since $B$ is a covering element of $A$, there are three possible cases for $B$ :

- If $B$ is $A_{1}-A_{2}-\cdots-A_{i} x-A_{k}$, then $C$ can get forms

- $A_{1}-A_{2}-\cdots-A_{i} x y-A_{k}$, or

- $A_{1}-A_{2}-\cdots-A_{i} x-A_{j} y-A_{k}$, or

- $A_{1}-A_{2}-\cdots-A_{i} x-A_{k}-y z$, or 
- $A_{1}-A_{2}-\cdots-A_{i} x-A_{j} \cup A_{l}-A_{k}$, or

- $A_{1}-A_{2}-\cdots-A_{i} x \cup A_{j}-A_{k}$.

. If $B$ is $A_{1}-A_{2}-\cdots-A_{i}-A_{k}-x y$, then $C$ can get the following forms

$$
\begin{aligned}
& \cdot A_{1}-A_{2}-\cdots-A_{i}-A_{k}-x y-z t, \\
& \cdot A_{1}-A_{2}-\cdots-A_{i}-A_{k}-x y z, \\
& \cdot A_{1}-A_{2}-\cdots-A_{i} z-A_{k}-x y, \\
& \cdot A_{1}-A_{2}-\cdots-A_{i} \cup A_{j}-A_{k}-x y, \\
& \cdot A_{1}-A_{2}-\cdots-A_{i} \cup x y-A_{k} .
\end{aligned}
$$

- For the final cases, when $B$ is $A_{1}-A_{2}-\cdots-A_{i} \cup A_{j}-A_{k}$, then $C$ will have 5 forms. Those are

$$
\begin{aligned}
\cdot & A_{1}-A_{2}-\cdots-A_{i} \cup A_{j}-A_{l} x-A_{k}, \\
\cdot & A_{1}-A_{2}-\cdots-A_{i} \cup A_{j} x-A_{k}, \\
\text { - } & A_{1}-A_{2}-\cdots-A_{i} \cup A_{j}-\cdots-A_{h} \cup A_{l}-A_{k}, \\
\cdot & A_{1}-A_{2}-\cdots-A_{i} \cup A_{j} \cup A_{l}-\cdots-A_{k}, \text { and } \\
\cdot & A_{1}-A_{2}-\cdots-A_{i} \cup A_{j}-\cdots-A_{k}-x y .
\end{aligned}
$$

In all cases, we find another covering element $B_{1}$ of $A$, and $B_{1}<C$. It is obvious that $B_{1}<M$. Then find $\lambda\left(A, B_{1}\right)$. We choose $B_{1}$ such that $\lambda\left(A, B_{1}\right) \leq \lambda(B, C)$, or the first component of $\lambda\left(A, B_{1}\right)$ equals the first component of $\lambda(B, C)$. Since $B$ covers $A$ in $\mathcal{C}$ and by the rule of choosing, we have $\lambda(A, B)<\lambda\left(A, B_{1}\right)$. Therefore, $\lambda(A, B)<\lambda(B, C)$.

We proved that $\mathcal{C}$ is an increasing chain. To prove $\lambda$ is an EL-labeling, we also need to show that $\mathcal{C}$ is the unique increasing maximal chain from $N$ to $M$.

Lemma 3.3. The increasing maximal chain from $N$ to $M$ is unique.

Proof. The main idea of the proof is getting a unique covering element $N_{1}$ of $N$ in $\mathcal{C}$. If $N_{1}^{\prime}$ is another covering element of $N$ in $[N, M]$, we try to show that the maximal chain $N \lessdot N_{1}^{\prime} \lessdot \cdots \lessdot M$ is not increasing by indicating one labeling value in the interval $\left[N_{1}^{\prime}, M\right]$ which is less than $\lambda\left(N, N_{1}^{\prime}\right)$. That indication depends on the form of $N_{1}$ and $N_{1}^{\prime}$. There are nine cases to consider and we omit straightforward proof.

Theorem 3.4. The labeling $\lambda$ is an EL-labeling.

Proof. By Lemma 3.2 and Lemma 3.3, we can see there is only one increasing maximal chain $\mathcal{C}$ from $N$ to $M$. By the rule we choose an increasing chain, it is easy to see that $\mathcal{C}$ lexicographically precedes all other maximal chains of $[N, M]$. Therefore, the labeling $\lambda$ is an $E L$ labeling. 
Example 3.5. Consider the intersection poset $P_{G_{4}}$ of $G_{4}$ in Figure 3. We use the above way to find an increasing chain $\mathcal{C}$ from 12 to 12345. Firstly, we find the covering element of 12 . There are three elements in $P_{G}$ covering 12 , and the labeling value $\lambda(12,123)=(3,1), \lambda(12,124)=$ $(4,1)$ and $\lambda(12,12-45)=5$. Since $\lambda(12,123)$ is the smallest value, we choose 123 for the covering element of 12 in $\mathcal{C}$. Next, we find the covering element of 123 in $\mathcal{C}$. We see that 123 is covered by 1234 and $123-45$, and the labeling value $\lambda(123,1234)=(4,1), \lambda(123,123-45)=5$. We must choose 1234 for the covering of 123 in $\mathcal{C}$. Finally, we choose 12345 for the covering of 1234 in $\mathcal{C}, \lambda(1234,12345)=(5,4)$. Obviously, $\mathcal{C}$ is an increasing chain.

We proved that the edge-labeling above is an EL-labeling. Theorem 2.8 and Theorem 3.4 imply the following corollary.

Corollary 3.6. The order complex $\Delta\left(\overline{P_{G}}\right)$ has the homotopy type of a wedge of spheres.

Since $P_{G}$ is the intersection poset of a hyperplane arrangement, all maximal chains in $P_{G}$ have the same cardinality [8, Lemma 2.3]. Therefore, the decreasing maximal chains of $P_{G}$ have the same length, and hence $\Delta\left(\overline{P_{G}}\right)$ has the homotopy type of a wedge of spheres of the same dimension. That dimension is also the length of $\overline{P_{G}}$ or $l\left(P_{G}\right)-2$ where $l\left(P_{G}\right)$ is the length of $P_{G}$. Let $c(G)$ be the number of connected components in $G$. It is not difficult to see that the length of $P_{G}$ is equal to $n-c(G)$. Therefore, the dimension of spheres in the wedge is equal to $n-c(G)-2$.

We already showed the existence of an increasing maximal chain in a closed interval $[N, M]$. Now we consider the existence of a decreasing maximal chain in that interval.

Lemma 3.7. There is a decreasing maximal chain from $N$ to $M$.

Proof. Let $\mathcal{D}$ be the decreasing maximal chain we need to find. We choose the covering element $X_{1}$ of $N$ in $\mathcal{D}$ if

$$
\lambda\left(\left\{N, X_{1}\right\}\right)=\max \{\lambda(\{N, X\}) \mid N \lessdot X<M\} .
$$

We continue to choose $X_{2}$ for covering element of $X_{1}$ in $\mathcal{D}$ if

$$
\lambda\left(\left\{X_{1}, X_{2}\right\}\right)=\max \left\{\lambda\left(\left\{X_{1}, X\right\}\right) \mid X_{1} \lessdot X<M\right\} .
$$

Use this way to find other elements in the maximal chain $\mathcal{D}$ until we get $M$.

Now we claim that $\mathcal{D}$ is a decreasing chain. 
Let $A, B$, and $C$ be three elements in the maximal chain $\mathcal{D}$ such that $A \lessdot B \lessdot C$. There are fifteen cases to consider which are shown in the proof of Lemma 3.2. In all cases, the common way to prove $\lambda(\{A, B\})>\lambda(\{B, C\})$ is also similar to the way used to prove the chain $\mathcal{C}$ is increasing. But the difference is choosing $B_{1}$ such that $\lambda\left(\left\{A, B_{1}\right\}\right) \geq \lambda(\{B, C\})$ or the first component of $\lambda\left(\left\{A, B_{1}\right\}\right)$ equals the first component of $\lambda(\{B, C\})$. Since $B$ covers $A$ in $\mathcal{D}$ and by the rule of choosing, we have $\lambda(\{A, B\})>\lambda\left(\left\{A, B_{1}\right\}\right)$. Therefore, $\lambda(\{A, B\})>\lambda(\{B, C\})$.

Remark 3.8. It is easy to see that a compressed graph is a chordal graph and Stanley mentions in [9] that a chordal graph is a supersolvable graph. Therefore, the intersection poset of the graphical arrangement of a chordal graph is a supersolvable lattice. Stanley also gives an edgelabeling on supersolvable lattice, and proves that it is an $E L$-labeling (see [9]). The labeling introduced in this paper can be easily obtained from the graph and is different from the labeling by Stanley. For example, we consider two labelings for the intersection poset of the graphical arrangement of the complete graph $K_{4}$ so that their unique increasing chains are the same. Then $\hat{0}<34<134<1234$ is a decreasing chain in Stanley's labelling while it is not decreasing in this paper's labelling.

\section{The number of decreasing maximal chains}

In this section, we will find the number of decreasing maximal chains of $P_{G}$, where $G$ is a compressed graph.

Let $\mathcal{M}:=\hat{0} \lessdot X_{1} \lessdot X_{2} \lessdot \cdots \lessdot X_{l} \lessdot \hat{1}$ be a maximal chain in $[\hat{0}, \hat{1}]$. We associate $\mathcal{M}$ with the ordered string

$$
\lambda(\mathcal{M})=\left[\lambda\left(\hat{0}, X_{1}\right), \lambda\left(X_{1}, X_{2}\right), \ldots, \lambda\left(X_{l}, \hat{1}\right)\right] .
$$

Since $P_{G}$ is the intersection poset of a hyperplane arrangement, the labeling values $\lambda\left(\hat{0}, X_{1}\right), \lambda\left(X_{1}, X_{2}\right), \ldots, \lambda\left(X_{l}, \hat{1}\right)$ are corresponding to hyperplanes or edges of $G$. The labeling values are based on the corresponding hyperplanes or edges of $G$. If the intersections of a subspace $S$ and different hyperplanes give us the same common subspace $T$, then the labeling value $\lambda(S, T)$ is found by using the hyperplane with smaller indices. For example, consider the maximal chain $\mathcal{M}=$ $\hat{0} \lessdot 12 \lessdot 123 \lessdot 1234 \lessdot 12345=\hat{1}$ in Figure 3 . We associate $\mathcal{M}$ with the ordered string of labeling values $\lambda(\mathcal{M})=[2,(3,1),(4,1),(5,4)]$. Whenever we get an intersection of a subspace and a new hyperplane, we also 
get a labeling value. So we can see that the labeling value 2 is corresponding to the hyperplane $x_{1}-x_{2}=0$ or the edge 12 . Similarly, the labeling values $(3,1),(4,1)$, and $(5,4)$ are corresponding to the edges 13 , 14 , and 45 respectively. We can associate the string of labeling values $\lambda(\mathcal{M})=[2,(3,1),(4,1),(5,4)]$ with the string of edges $[12,13,14,45]$. In other words, we can associate the maximal chain $\mathcal{M}$ with the string of edges $[12,13,14,45]$.

The following lemma, whose straightforward proof is omitted, gives the condition when an edge is corresponding to a labeling value.

Lemma 4.1. Let $X$ be a string of edges of a compressed graph $G$, and $x$ be an edge in $X$. Then $x$ is corresponding to a labeling value (or used to find a labeling value) if and only if both of the following situations do not happen:

I. Both vertices of $x$ already appear in $X$ before the edge $x$ and there is another edge $y$ in $G$ satisfying

- there exists a cycle containing $x$ and $y$ such that all the other edges appear in $X$ before $x$,

- $(e, f)<(a, b)$ if $x=a b(a<b)$ and $y=e f(e<f)$.

II. One of the vertices of $x$, say $a$, already appear in $X$ before $x$ and there exists another edge $y=b e$ in $G$ containing the other vertex $b$ of $x$ satisfying

- there exists a cycle containing $x$ and $y$ such that all the other edges appear in $X$ before $x$,

. $e<a$.

A spanning forest of a graph $G$ is a union of spanning trees in all connected components of $G$.

Definition 4.2. Let $X$ be a string of edges of a graph $G$. We say $X$ is associated with a maximal chain if

- The edges in $X$ form a spanning forest of $G$.

- Each edge in $X$ gives us a labeling value.

- The number of edges in the string is the length of poset $P_{G}$.

Furthermore, $X$ is associated with a decreasing maximal chain if the labeling values corresponding to the edges in $X$ are decreasing.

Given an arbitrary string of edges of length $l\left(P_{G}\right)$, we cannot say that it is associated with a maximal chain of $P_{G}$. Also, if we change the order of edges in the string associated with a maximal chain, it can be not related to any maximal one. On the other hand, we also can rearrange edges in a string of edges to associate it with a maximal chain 


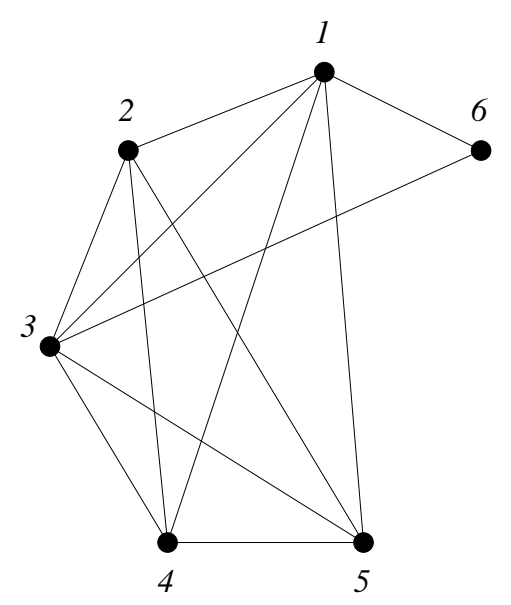

Figure 4. A compressed graph $G_{5}$

or a decreasing maximal chain. The following examples will show us this issue.

Example 4.3. See Figure 4 for another compressed graph. Let $X=$ $[16,23,24,25,12]$ be a string of edges. The edges in $X$ form a spanning forest of $G_{5}$. Furthermore, $X$ is associated with the string of labeling values $[6,3,(4,2),(5,2),(1,2)]$ or the maximal chain $\hat{0} \lessdot 16 \lessdot 16-23 \lessdot$ $16-234 \lessdot 16-2345 \lessdot 123456$. Now we change the order of edges in $X$ to get the string $X^{\prime}=[16,12,23,24,25]$. In order to see whether $X^{\prime}$ is associated with a maximal chain or not, we have to check whether all the edges are used to find labeling values. Firstly, edge 16 is used to find the labeling value when we get the intersection of hyperplane 16 and $\mathbb{R}^{6}$, and the corresponding labeling value $\lambda(\hat{0}, 16)=6$. Since the intersection of subspace 16 and hyperplane 12 gives us the subspace 126 and the labeling value $\lambda(16,126)=(2,1)$, the edge 12 is also used to find a labeling value. Now we consider the intersection of 126 and hyperplane 23. This intersection gives us subspace 1236, and the labeling value $\lambda(126,1236)=(3,1)$. Therefore, the edge 23 is not used to find a labeling value in this case. Hence $X^{\prime}$ is not associated with a maximal chain.

The following lemma provides some conditions for a string of edges to be associated with a decreasing maximal chain. 
Lemma 4.4. Let $X=\left[x_{1}, x_{2}, \ldots, x_{l}\right]$ be a string of edges of a compressed graph $G$ where $l$ is the length of the poset $P_{G}$. Then, the edges in $X$ form a spanning forest of $G$ and there do not exist any two edges $a b$ and $c b$ in $X$ where $a, c<b$ if and only if the edges in $X$ can be rearranged to get a new string $X^{\prime}$ associated with a decreasing maximal chain in $[\hat{0}, \hat{1}]$. Furthermore, $X^{\prime}$ is unique.

Proof. Let $X=\left[x_{1}, x_{2}, \ldots, x_{l}\right]$ be a string of edges satisfying the given conditions. Since the edges in $X$ form a spanning forest of $G$, we can find a path using edges in $X$ to connect any two vertices in a connected component of $G$.

Suppose there exist edges $a b$ and $c b$ in $X$ where $a, c<b$. Since $G$ is compressed, $G$ must have the edge $a c$. Since any labeling value obtained from $a c$ is less than any labeling value got by edges $a b$ or $c b$, the latter of $a b$ and $c b$ in $X$ does not correspond to a labeling value. Thus there is no rearrangement of edges in $X$ associated with a decreasing maximal chain.

Now assume that $X$ does not have edges $a b$ and $c b$ such that $a, c<$ $b$. Then we can rearrange edges in $X$ to get a new string $X^{\prime}$ that is associated with a decreasing maximal chain by the following rule.

Let $m$ be the largest non-isolated vertex of $G$. By the given condition of $X, m$ appears in only one edge, say $x m(x<m)$. We choose $x m$ for the first position in the string $X^{\prime}$.

Assume that we already have edges $x_{1}, x_{2}, \ldots, x_{k}$ in $X^{\prime}$. Then there are three cases:

(1) If $\left\{\right.$ vertices of $\left.x_{1}, x_{2}, \ldots, x_{k}\right\} \neq[n]$ and there is an edge in $X$ both of whose vertices appear in $x_{1}, \ldots, x_{k}$ and greater than $\max ([n] \backslash\{$ vertices of $\left.\left.x_{1}, x_{2}, \ldots, x_{k}\right\}\right)$, then we add an edge $x=a b(a<b)$ where $(a, b)$ is maximal among those edges.

(2) If $\left\{\right.$ vertices of $\left.x_{1}, x_{2}, \ldots, x_{k}\right\} \neq[n]$ and there does not exist any edge in $X$ such that both of whose vertices appear in $x_{1}, \ldots, x_{k}$ and greater than $\max \left([n] \backslash\left\{\right.\right.$ vertices of $\left.\left.x_{1}, x_{2}, \ldots, x_{k}\right\}\right)$, then we add an edge $x=a b$ where $b$ is the maximal new vertex and $a$ is maximal among the vertices adjacent to $b$.

(3) If $\left\{\right.$ vertices of $\left.x_{1}, x_{2}, \ldots, x_{k}\right\}=[n]$, then we add an edge $x=a b(a<$ $b)$ where $(a, b)$ is maximal among the remaining edges.

We see that an edge $a b$ appears before an edge $c d$ in $X^{\prime}$ when there is at least one vertex in $a b$ that is greater than one vertex in $c d$.

Now we claim that every edge in $X^{\prime}$ is corresponding to a labeling value. Suppose that there is an edge $x=a b(a<b)$ in $X^{\prime}$ not corresponding to a labeling value. There are two cases from Lemma 4.1. 
I. In this case, there exist an edge $y=e f(e<f)$ of $G \backslash X$ and a cycle containing $x$ and $y$. Also, note that $e<f<a<b$ and all the edges of the cycle except $x$ and $y$ appear in $X^{\prime}$ before $x$. There are two sub cases:

(a) When $e$ is closer to $b$ than to $a$ in the cycle:

In this case, the vertices in the path from $b$ to $e$ in the cycle must be increasing because of the condition of $X$. But this implies $b<e$ which is impossible.

(b) When $e$ is closer to $a$ than to $b$ in the cycle:

In this case, the vertex adjacent to $a$ in the cycle must be bigger than $a$ since the edge connecting this vertex and $a$ appears before $x$ in $X^{\prime}$ by the construction of $X^{\prime}$. Then the path from $a$ to $e$ is increasing which implies $a<e$. This is also impossible.

II. In this case, there is an edge $y$ in $G \backslash X$ adjacent to $x$ and a cycle containing $x$ and $y$. Also note that the vertex of $y \backslash x$ must be smaller than the vertex in $x \backslash y$ and all the edges of the cycle except $x$ and $y$ appear in $X^{\prime}$ before $x$. We omit the proof for this case which is similar to the previous case.

It is obvious that the choosing gives us a decreasing chain. In each step of choosing, we have only one choice. So we can get only one string $X^{\prime}$ from $X$ that is associated with a decreasing maximal chain.

Example 4.5. We use the compressed graph $G_{5}$ shown in Figure 4 . Let $X=[12,13,34,36,45]$ be a string of edges of $G_{5}$. The edges in $X$ form a spanning forest of $G_{5}$. Now we use the rule above to find a new string $X^{\prime}$ that can be associated with a decreasing maximal chain. Firstly, we choose 36 for the first position in $X^{\prime}$. Since $5=\max \{[6] \backslash\{3,6\}\}$ and there is only one edge containing 5 in $X$, we choose 45 for the next position. Although $2=\max \{[6] \backslash\{3,6,4,5\}\}$, there is an edge 34 that has $2<3<4$. We add 34 into the next position of $X^{\prime}$. After that, 12 is added into $X^{\prime}$. Finally, we add 13 to combine blocks. The new string $X^{\prime}=[36,45,34,12,13]$, and the string of labeling values is $[6,5,(3,4), 2,(1,3)]$. It is associated with the decreasing maximal chain $\hat{0} \lessdot 36 \lessdot 36-45 \lessdot 3456 \lessdot 12-3456 \lessdot 123456$.

Now consider adjacent edges of vertices of $G$. Let

$$
\alpha(i)=\{x i \mid x i \in E(G), x<i\}
$$

for all $i=2, \ldots, n$. 
Theorem 4.6. The number of decreasing maximal chains of the intersection poset $P_{G}$ is

$$
\prod_{i=2,|\alpha(i)| \neq 0}^{i=n}|\alpha(i)|
$$

where $|\alpha(i)|$ is the number of edges in $\alpha(i)$ for $i=1,2, \ldots, n$. Furthermore, the order complex $\Delta\left(\overline{P_{G}}\right)$ has the homotopy type of a wedge of $\prod_{i=1,|\alpha(i)| \neq 0}^{i=n}|\alpha(i)|$ spheres of dimension $n-c(G)-2$, where $c(G)$ is the number of connected components in $G$.

Proof. Lemma 3.7 shows that there is a string $X$ of edges that is associated with a decreasing maximal chain.

Let $X=\left[x_{1}, x_{2}, \ldots, x_{l-1}, x_{l}\right]$ be a string of edges that can be associated with a decreasing maximal chain. By Lemma 4.4, $X$ does not contain two edges $a b$ and $c b$ such that $a, c<b$, and the number of edges in $X$ is equal to the length, $n-c(G)$, of $P_{G}$. It is easy to see that the vertices $v$ with $|\alpha(v)|=0$ are precisely the vertices with the smallest label in each connected component of $G$. So the length of $P_{G}$ (or the number of edges in $X$ ) is equal to the number of vertices $v$ that have $|\alpha(v)| \neq 0$.

In other words, if $|\alpha(v)| \neq 0$, then there is only one edge in $X$ whose larger vertex is $v$.

Let $g m(g<m)$ be an edge of $X$ where $m$ is the largest vertex appear in $X$. Since the set $X^{\prime}=X \backslash\{g m\} \cup\left\{g^{\prime} m\right\}$, where $g^{\prime}<m$, satisfies the condition for Lemma 4.4, we can rearrange the edges in $X^{\prime}$ to get another maximal chain. There are $|\alpha(m)|$ ways to do this. Since the smaller vertices in other edges in $X$ can be replaced in similar way, the number of decreasing chains in $P_{G}$ is

$$
\prod_{i=1,|\alpha(i)| \neq 0}^{i=n}|\alpha(i)|
$$

The second part of the theorem follows from Theorem 2.8.

Example 4.7. Let $G$ be a compressed graph as Figure 4. Use Theorem 4.6 , we can indicate that the order complex $\Delta\left(\overline{P_{G}}\right)$ has the homotopy type of a wedge of 48 spheres of dimension 3 .

Corollary 4.8. The order complex $\Delta\left(\overline{P_{K_{n}}}\right)$ has the homotopy type of a wedge of $(n-1)$ ! spheres of dimension $n-3$, where $K_{n}$ is a complete graph. 
Proof. It is easy to see that $|\alpha(i)|=i-1$ for all $i=1,2, \ldots, n$. Theorem 4.6 indicates that the number of decreasing maximal chains in $P_{K_{n}}$ is $(n-1)$ !. Therefore, the order complex $\Delta\left(\overline{P_{K_{n}}}\right)$ has the homotopy type of a wedge of $(n-1)$ ! spheres of dimension $n-3$.

Corollary 4.9. If $G$ is a compressed graph with no cycle, then there is only one decreasing maximal chain from $\hat{0}$ to $\hat{1}$. Furthermore, the order complex $\Delta(\bar{P})$ has the homotopy type of a sphere of dimension $n-c(G)-2$, where $c(G)$ is the number of connected components of $G$.

Proof. If $G$ is a compressed graph with no cycle, then it is obvious that $|\alpha(i)|=0$ or 1 for all vertex $i$. Therefore, the number of decreasing maximal chains in $P_{G}$ is 1 .

The next lemma will give some conditions to help us find the number of decreasing maximal chains of $P_{G}$, where $G$ is a compressed graph with one cycle.

Lemma 4.10. If $G$ is a compressed graph with only one cycle, then the cycle must be a 3 -cycle with vertices $a, b$, and $b+1$ for some $a<b$.

Proof. Assume that the cycle has more than 3 edges. Let $i_{1} i_{2}$ be an edge of the cycle such that $i_{1}<i_{2}$ and $\left|i_{1}-i_{2}\right|=\max \left\{\left|i_{x}-i_{y}\right|: i_{x} i_{y} \in\right.$ $E(G)\}$.

Firstly, we claim that there exists $i_{3}$ in the cycle such that $i_{1}<i_{3}<$ $i_{2}$. Suppose there is no such vertex in the cycle. Then one can label the vertices of the cycle so that $i_{k} i_{k+1}$ are edges for $k=1, \ldots, m-1$ and $i_{m} i_{1}$ is an edge. One can see that $i_{k}>i_{2}$ for all $k=3, \ldots, m$ because of the choice of $i_{1} i_{2}$. But then $\left|i_{m}-i_{1}\right|>\left|i_{1}-i_{2}\right|$ which is impossible.

Since $G$ is compressed, it has the edge $i_{1} i_{3}$. Since $G$ has only one cycle, $i_{1} i_{3}$ must be contained in a cycle. Now suppose $i_{2}$ and $i_{3}$ are not adjacent. Let $j$ be the vertex adjacent to $i_{3}$ in the cycle. We can choose edge $i_{3} j$ since the cycle has more than 3 edges. We get four cases, $j<i_{1}<i_{3}<i_{2}$ or $i_{1}<j<i_{3}<i_{2}$, or $i_{1}<i_{3}<j<i_{2}$, or $i_{1}<i_{3}<i_{2}<j$. In all four cases, $G$ also has more than one cycle. Therefore, the unique cycle of $G$ must be a 3 -cycle.

Furthermore, assume that there is $d$ such that $a<b<d<c$, then we get edges $a d$ and $b d$ and so $G$ will have more than one cycle. So $c=b+1$.

Corollary 4.11. If $G$ is a compressed graph with one cycle, then the order complex $\Delta\left(\overline{P_{G}}\right)$ has the homotopy type of a wedge of two spheres. 
Proof. If the vertices of the unique cycle of $G$ are $a, b$, and $b+1$, then $|\alpha(b+1)|=2$ and $|\alpha(i)|=0$ or 1 for all $i \neq b+1$. Therefore,

$$
\prod_{i=1,|\alpha(i)| \neq 0}^{i=n}|\alpha(i)|=2
$$

and the order complex $\Delta\left(\overline{P_{G}}\right)$ has the homotopy type of a wedge of two spheres.

\section{References}

[1] Anders Björner, Shellable and Cohen-Macaulay partially ordered sets, Trans. Amer. Math. Soc., 260(1) (1980), 159-183.

[2] Anders Björner and Michelle Wachs, On lexicographically shellable posets, Trans. Amer. Math. Soc., 277(1) (1983), 323-341.

[3] Anders Björner and Michelle L. Wachs, Shellable nonpure complexes and posets. I, Trans. Amer. Math. Soc., 348(4) (1996), 1299-1327.

[4] Anders Björner and Michelle L. Wachs, Shellable nonpure complexes and posets. II, Trans. Amer. Math. Soc., 349(10) (1997), 3945-3975.

[5] Curtis Greene and Thomas Zaslavsky, On the interpretation of Whitney numbers through arrangements of hyperplanes, zonotopes, non-Radon partitions, and orientations of graphs, Trans. Amer. Math. Soc., 280(1) (1983), 97-126.

[6] Dmitry Kozlov, Combinatorial algebraic topology, volume 21 of Algorithms and Computation in Mathematics, Springer, Berlin, 2008.

[7] Peter Orlik and Louis Solomon, Combinatorics and topology of complements of hyperplanes, Invent. Math., 56(2) (1980), 167-189.

[8] Peter Orlik and Hiroaki Terao, Arrangements of hyperplanes, volume 300 of Grundlehren der Mathematischen Wissenschaften [Fundamental Principles of Mathematical Sciences], Springer-Verlag, Berlin, 1992.

[9] R. P. Stanley, Supersolvable lattices, Algebra Universalis, 2 (1972), 197-217.

[10] Richard P. Stanley, An introduction to hyperplane arrangements, In Geometric combinatorics, volume 13 of IAS/Park City Math. Ser., pages 389-496. Amer. Math. Soc., Providence, RI, 2007.

[11] Michelle L. Wachs, A basis for the homology of the d-divisible partition lattice, Adv. Math., 117(2) (1996), 294-318.

[12] Michelle L. Wachs, Poset topology: tools and applications, In Geometric combinatorics, volume 13 of IAS/Park City Math. Ser., pages 497-615. Amer. Math. Soc., Providence, RI, 2007.

[13] Thomas Zaslavsky, Facing up to arrangements: face-count formulas for partitions of space by hyperplanes, Mem. Amer. Math. Soc., 1(issue 1, 154):vii+102, 1975.

Thi A. Nguyen

Department of Mathematics, Chonnam National University, 
Gwangju 500-757, Republic of Korea.

E-mail : thinguyen16@gmail.com

\section{Sangwook Kim}

Department of Mathematics, Chonnam National University,

Gwangju 500-757, Republic of Korea.

E-mail : swkim.math@chonnam.ac.kr 